\title{
Performance and Analysis of MIMO Multi Channel Beam forming Systems under the Double Scattering Channel
}

\author{
A.Sanyasi Rao ${ }^{1}$ and G.Naveen Kumar $^{2}$
}

Abstract-We present the new method to investigate and analyze the symbol error rate performance of the multiple-input multiple-output (MIMO) multi-channel beam forming (MB) under the doublescattering channel. In the proposed method we derive an asymptotic expansion on the marginal eigen-value distribution of the MIMO channel matrix, and use the result to obtain an approximate expression on the average SER at high signal-to-noise ratio (SNR). Two major parameters of the SER, i.e., the diversity gain and the coding gain are analyzed. Our simulation results explain that it suffices for the double-scattering channel to have only limited scatterers, if the same diversity gain as the Rayleigh channel is desired; however, when the number of scatterers is below a certain level, the coding gain in the double-scattering channel will vary with the SNR logarithmically.

Keywords -Double-scattering channel, eigen-value distribution, diversity gain, coding gain.

\section{INTRODUCTION}

The prospect of extraordinary improvements in the capacity of wireless networks has drawn considerable attention to multiple-input-multiple-output (MIMO) communication techniques. MIMO methods make use of multi-element antenna coding at both the transmit and the receive side of a radio link to drastically improve the capacity over more traditional single-input-multiple-output (SIMO) systems. Multiple-input multiple-output (MIMO) multichannel beam-forming (MB) [3] a linear transmission scheme which applies perfect channel state information (CSI) at the transmitter and receiver to guide multiple data streams along the strongest eigen-direction of the MIMO channel.

\section{SYSTEM MODEL AND PROBLEM FORMULATION MIMO MB System Model}

We consider a MIMO wireless communication system having $\mathrm{nT}$ transmit and $\mathrm{nR}$ receive antennas. The received vector is given is given by

$Y=H X+n$

\section{ASYMPTOTIC EXPANSION OF THE EIGENVALUE DISTRIBUTION}

First of all we present the exact expression on the Eigen value distribution of HHH. Based on the exact distribution, we then derive its asymptotic expansion. For notational convenience,

we define through the rest of this paper: $S \triangleq \min \left(\mathrm{n}_{\mathrm{R}}, \mathrm{n}_{\mathrm{S}}\right), T \triangleq$

\footnotetext{
${ }^{1}$ Dept of ECE, Balaji Institute Of Technology \& Sciences College

${ }^{2}$ Dept of ECE, Balaji Institute Of Technology \& Sciences College
} 
with $K v(\cdot)$ being the modified Bessel function of the second kind

To see the complexity of the exact SER result, we substitute back into and get an expression consisting of special functions, determinants, and integrals. Knowing this, we turn our attention to the approximate SER .Our first step is to derive e asymptotic expansion on the eigen-value distribution, but unfortunately, we find that the conventional deriving technique not applicable here. This conventional technique, termed as differential-based method requires the function to be expanded) to be differentiable around zero. However, the CDF here is not even continuous around zero (as the modified Bessel function $K(\cdot)$ is discontinuous at the origin for $v \in Z$ ). A deriving technique that applies for double scattering channels of arbitrary configurations $(\mathrm{nR}, \mathrm{nS}, \mathrm{nT})$ is needed.

\section{PROPOSED METHOD}

In this context, we propose here the Expand Remove-Omit method, which does not require the differentiability of the $\mathrm{CDF}$, and, more importantly, is applicable to arbitrary double-scattering channels.

2) Expand the $\alpha i$-th column of the matrix $\Delta$ using the multi-linear property of the determinant (see below), and get multiple matrices with exponential terms of different orders (let $\hbar \mathrm{i}, \mathrm{j}(\cdot)$ denote a generic function, and "l" denote "except")

$$
\begin{aligned}
& \operatorname{det}\left\{\begin{array}{c}
\sum_{l} \hbar \mathrm{i}, \mathrm{j}(\mathrm{l}), \mathrm{i}=1 \ldots . \mathrm{n} ; \mathrm{j}=\mathrm{m} ; \\
a i, j, \mathrm{i}=1 \ldots . \mathrm{n} ; \mathrm{j}=\{1, \ldots . \mathrm{n}\} \backslash \mathrm{m} ;
\end{array}\right\} \\
& =\sum_{l}\left\{\begin{array}{c}
\hbar \mathrm{i}, \mathrm{j}(\mathrm{l}), \mathrm{i}=1 \ldots . \mathrm{n} ; \mathrm{j}=\mathrm{m} ; \\
a i, j, \mathrm{i}=1 \ldots . \mathrm{n} ; \mathrm{j}=\{1, \ldots . \mathrm{n}\} \backslash \mathrm{m} ;
\end{array}\right\}
\end{aligned}
$$

3) Remove matrices with co-linear columns as their determinants are zero-valued;

4) Omit other matrices, leaving only the one with the lowest-order exponential term; denote the remaining matrix as $\Delta \mathrm{i}$;

5) Let $i=i+1$;

6) If $i \leq M+1-k$, go back to 2); otherwise, continue;

7) If all permutations of $(\beta k, \ldots, \beta M)$ have been used, continue; otherwise, update $(\alpha 1, \ldots, \alpha M+1-k)$ with a new permutation of $(\beta k, \ldots, \beta M)$ and go back to 2 ;

8) Sum up determinants of the remaining matrices for all possible $(\alpha 1, \ldots,+1-k)$, and get the following equality:

$\operatorname{det}[\Delta 0]=\alpha 1, \ldots, \alpha \mathrm{M}+1-\mathrm{k} \operatorname{det}[\Delta \mathrm{M}+1-\mathrm{k}]+\mathcal{O}$, where $\mathcal{O}$ denotes the higher-order infinitesimal;

9) Factor out all exponential terms of $\operatorname{det}[\Delta 0]$ into $z d k$ minimize $d k$ over all possible $\beta$, and finally get the desired term $d k$. The remaining part of $\operatorname{det}[\Delta 0]$ after the factorization then equals $c k(z)$.

Detailed description of the method is in [1, App. A], here we present directly its expanding result.

Theorem 1. The marginal CDF $F \lambda k(z)$ can be expanded as

$(k=1, \ldots, \mathrm{M})$

$\mathrm{o}(z \mathrm{dk})$ is the higher-order infinitesimal of $z \mathrm{dk}$ as $z$ approaches zero, $\mathcal{C} k, i$ is a constant coefficient, and $\mathbb{S} k$ is a set of nonnegative integer numbers. Both $\mathbb{S} k$ and $\mathcal{C} k$, i are uniquely determined by[2,Eq. (21)].

\section{SYSTEM PERFORMANCE IN THE HIGH-SNR REGIME}

Now, we apply the asymptotic expansion to analyze the performance of MIMO MB in the high-SNR regime. We express the average SER of the -th strongest sub-stream as $(k=1 \ldots L)$ 
The average SERs of all the sub-streams are plotted in Fig.1, where each "Analytical SER" curve is

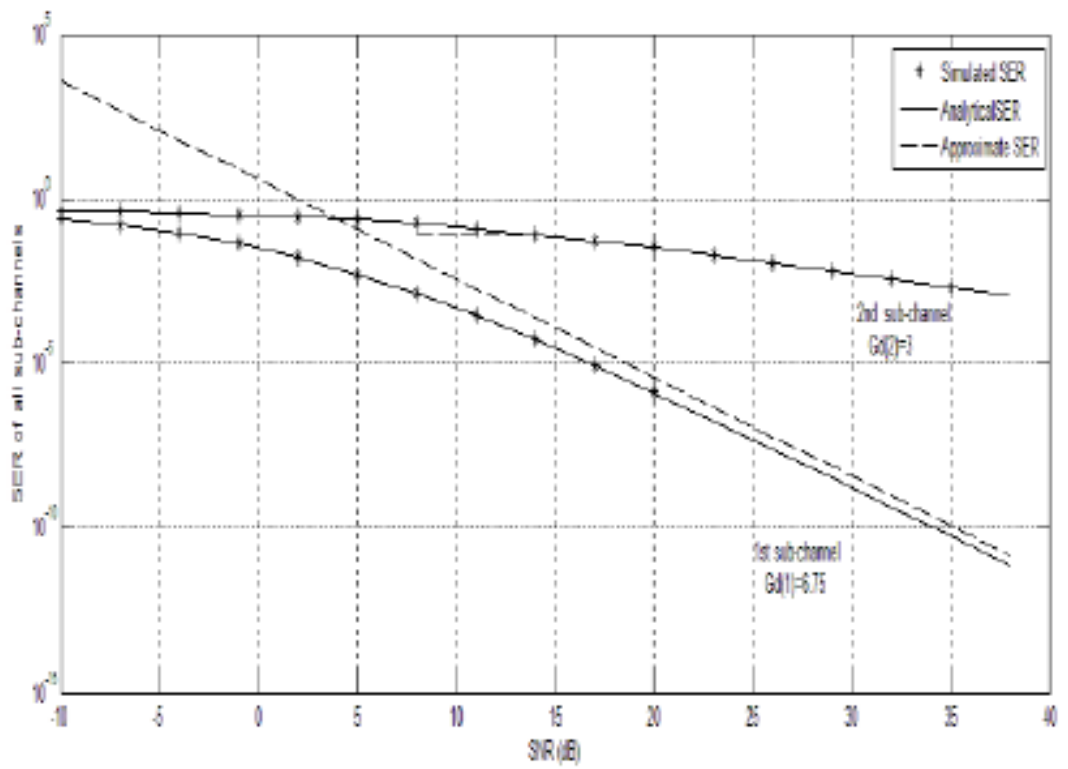

Fig.1. SER of all MIMO MB sub-streams in a $(3,3,3)$ double-scattering channel

computed by substituting into Clearly, we can see that two diversity gains, 6.75 and 3, are attained by the two sub-streams, respectively, which is in perfect agreement with our theoretical result . To see the impact of the scatterer on the diversity gain, we fix

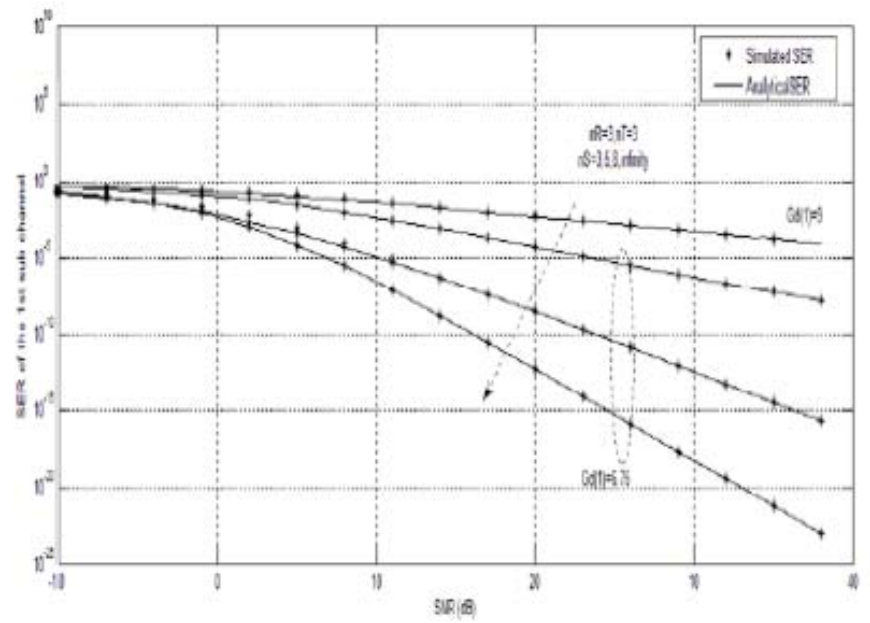

Fig. 2. SER of the strongest sub-stream in four double-scattering channels: $(3,3,3),(3,5,3),(3,8,3) \&(3, \infty, 3)$

Fig.2. SER of the strongest sub-stream in four double-scattering channels: $(3,3,3),(3,5,3),(3,8,3) \&(3, \infty$, 3) average SER of the strongest sub-stream is plotted in Fig. 2.In the $(3,3,3)$ case, we observe a diversity gain of 6.75, which is exactly the same as we expected from (18). In the remaining cases, we notice that, once the number of scatterer is greater than 5, adding more scatterers into the channel will not change the diversity gain. This verifies our earlier analysis that the diversity gain reaches its upper bound whenever $\mathrm{nS} \geq \mathrm{nR}+\mathrm{nT}-1$. 
Also from (18) we observe that the diversity gain is independent of the order of (nR, nS, nT).i.e, (nR, $\mathrm{nS}, \mathrm{nT}),(\mathrm{nR}, \mathrm{nT}, \mathrm{nS}),(\mathrm{nT}, \mathrm{nR}, \mathrm{nS}),(\mathrm{nT}, \mathrm{nS}, \mathrm{nR})$, and $(\mathrm{nS}, \mathrm{nR}, \mathrm{nT}),(\mathrm{nS}, \mathrm{nT}, \mathrm{nR})$, are indeed equivalent. This is an extension to the results of [4, Theo. 2] and [3, Theo. 4], where they showed that interchanging $\mathrm{nR}$ with $\mathrm{nT}$ would not change the diversity gain of the Rayleigh/ Rician channel. . Besides this rotational symmerty, we also see that the diversity gain of the $k$-th sub-stream in a (nR, nS, nT) channel is indeed equivalent to that of the first sub-stream in a $(\mathrm{nR}+1-k, \mathrm{nT}+1-k, \mathrm{nT}+1-k)$ channel. This indicates that reducing the sub-stream index by 1 is equivalent, by reducing the number of transmit antenna; receive antenna and scatterers all by one in the sense of diversity gain.

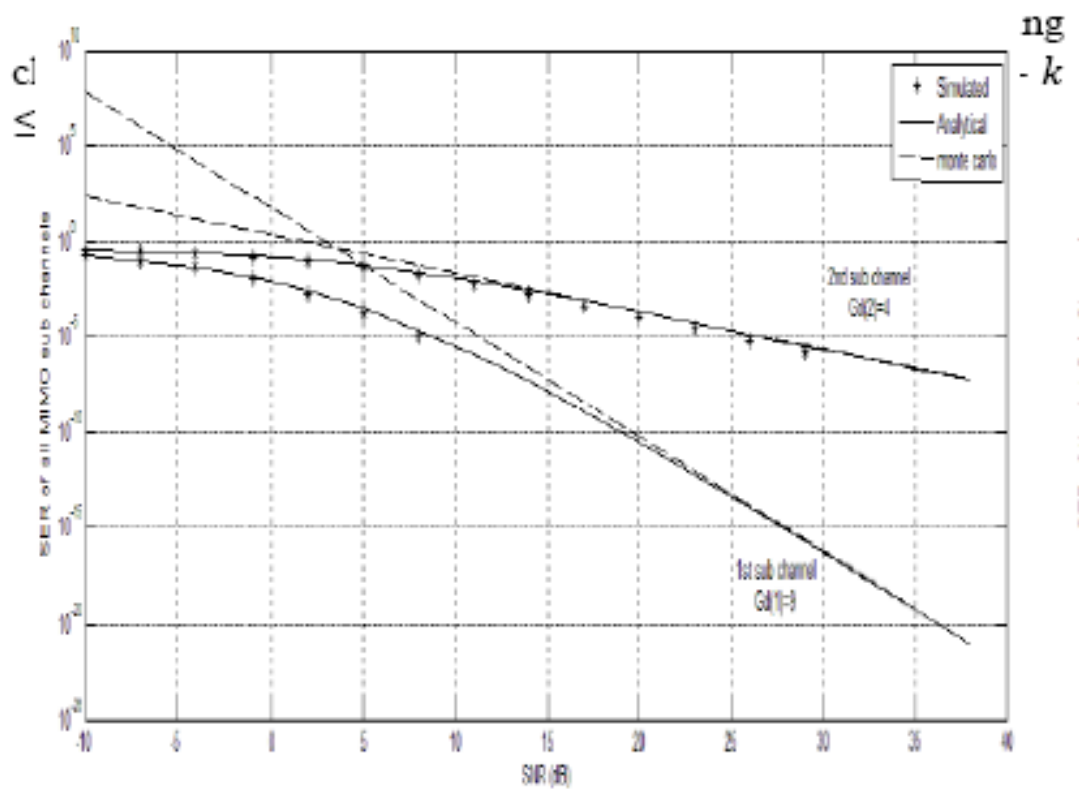

Fig.3. SER of all sub-streams in a $(3,3,4)$ double-scattering channel

else, it varies logarithmically with $\rho$. Our result here provides a whole picture of the coding gain. To verify our more general result, we present in Fig.3.the average SER of the MIMO MB

system in a $(3,3,4)$ double-scattering channel. (The "approximate SER" is computed based on Corollary 1).We can notice that the approximate SER results agree very well with actual curves especially in the high-SNR regime.

\section{CONCLUSION}

We have examined the average SER performance of MIMO Multichannel Beam forming under the general double-scattering channel Model.Our results are based on two perfo- rrmance parameters, i.e., the diversity gain and the coding gain, which characterized the SER of the system in the high SNR regime. In order to get analytical results on the two gains, we derived asymptotic expansions on the eigen-value distribution of the MIMO channel matrix, introducing a new method known as "ExpandRemove-Omit Method". The asymptotic expansion was then applied to get the approximate expression for the average SER. Our results proved that the diversity gain of the double scattering channel was upper bounded by the diversity gain of the corresponding Rayleigh 


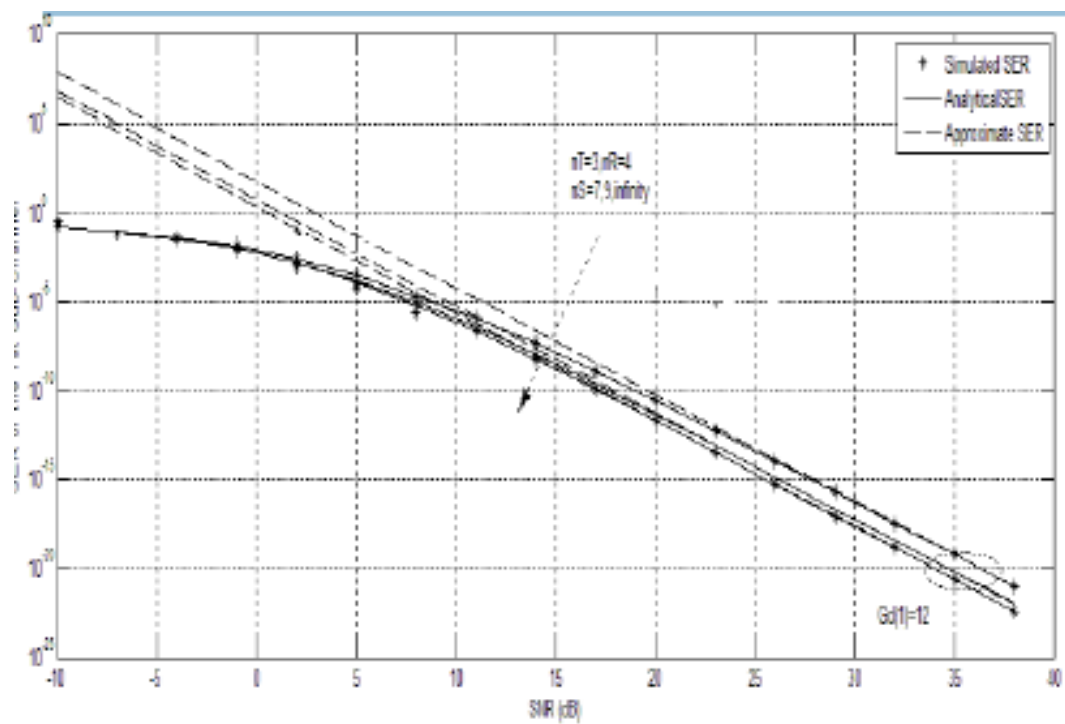

Fig.4. SER of the strongest sub-streams in three double-scattering channels: $(3,7,4),(3,9,4)$, and $(3, \infty, 4)$

when the condition $\mathrm{nS} \geq \mathrm{nR}+\mathrm{nT}-1$ was satisfied.Unlike conventional Rayleigh and Rician channels, where the coding gain was a constant number, we proved here that, the coding gain of the doublescattering channel was indeed a function of the SNR which becomes independent of SNR only when $\mathrm{nS}$ $\geq \mathrm{nR}+\mathrm{nT}$ is satisfied.

\section{REFERENCES}

[1] J. G. Proakis, Digital Communications, 4th edition. McGraw-Hill, 2001.

[2] Haochuan Zhang, Shi Jin, Matthew R. McKay, Xin Zhang, and Dacheng Yang” High-SNR Performance of MIMO Multi-Channel Beamforming in Double-Scattering Channels" IEEE Trans. Communication, Vol. 59, No. 6, , pp.1621-1631, June 2011

[3] S. Jin, M. R. McKay, X. Gao, and I. B. Collings, "MIMO multichannel beam forming: SER and outage using new eigenvalue distributions of complex noncentral Wishart matrices," IEEE Trans. Commun., vol. 56, no. 3, pp. 424-434, Mar. 2008.

[4] A. Zanella, M. Chiani, and M. Z. Win, "On the marginal distribution of the eigenvalues of Wishart matrices," IEEE Trans. Commun., vol. 57, no. 4, pp. 1050-1060, Apr. 2009.

[5] L. G. Ordóñez, D. P. Palomar, A. Pages-Zamora, and J. R. Fonollosa, "High-SNR analytical performance of spatial multiplexing MIMO sys- tems with CSI," IEEE Trans. Signal Process., vol. 55, no. 11, pp. 5447-5463, Nov. 2007.

[6] L. G. Ordóñez, D. P. Palomar, and J. R. Fonollosa, "Ordered eigenvalues of a general class of Hermitian random matrices with application to the performance analysis of MIMO systems," IEEE Trans. Signal Process., vol. 57, no. 2, pp. 672-689, Feb. 2009. 


\section{AUTHOR 1:-}

Allanki Sanyasi Rao, received his Bachelors Degree in Electronics \& Communication Engineering in 1997, Masters Degree in Digital Systems \& Computer Electronics in 2012. He is having 18 years of teaching experience. Currently he is Associate Professor at Balaji Institute of Technology\& Sciences, Warangal, India. His research interest includes Wireless Communications, Signal Processing and Network Security \& Cryptography.

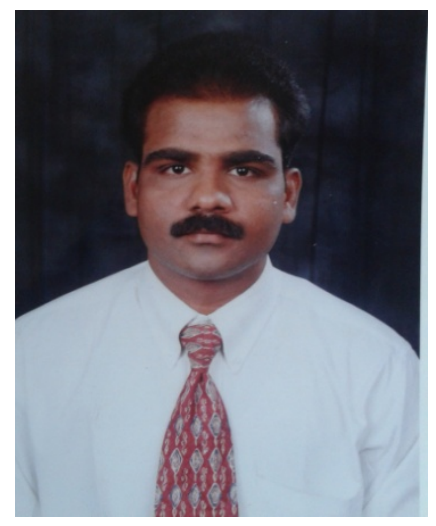

\section{AUTHOR 2:-}

Gandloju Naveen kumar completed his b.tech in balaji institute of engineering and sciences.and pursuing M.Tech in balaji institute of technology and sciences.

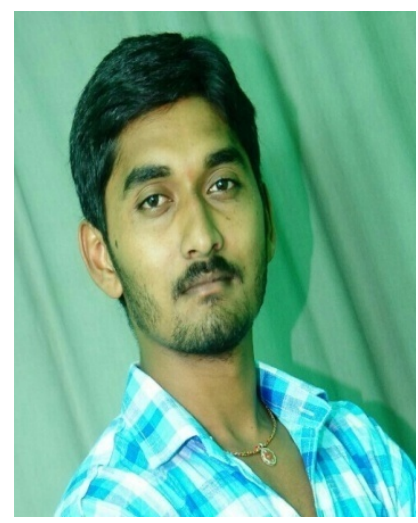

